\title{
Recent results of LEPS, ELPH and prospects of LEPS2
}

\section{Masayuki Niiyama for the LEPS2 collaboration*}

E-mail: nilyamadscphys.kyoto-u.ac.ip

Hadron photoproduction in a few $\mathrm{GeV}$ region has been studied at the SPring-8/LEPS and the ELPH, Tohoku University. The recent results are summarized in this article. LEPS and ELPH have collaborated to construct a new GeV-photon beamline at SPring-8 (LEPS2). The commissioning experiment utilizing a new photon detector, BGO-EGG, has started and is being ready for physics data taking. The detector development of a large solid angle solenoid magnetic spectrometer is underway. The current status of LEPS2 is also reported.

XV International Conference on Hadron Spectroscopy-Hadron 2013

4-8 November 2013

Nara, Japan

\footnotetext{
* Speaker.
} 


\section{Introduction}

The Research Center for Electron Photon Science at Tohoku University (ELPH) and the Laser Electron Photon facility at SPring-8 (LEPS) are the GeV photon facilities for hadron physics in Japan. These facilities provide the photon beam which has complementary characteristics, and various hadron photoproduction has been studied. In addition to physics studies, we have provided test beams for the detector development for high energy and nuclear physicists, for example, JPARC, Belle II, ATLAS and so on. The collaboration between ELPH and LEPS has been stronger since the Great East Japan Earthquake in 2011. ELPH and LEPS members have worked together to provide test beams which are important not only for physics studies but also for the education to students.

ELPH provides a high intensity photon beam of $10 \mathrm{Mcps}$ which is produced by the Bremsstrahlung process. The energy range is from 0.57 to $1.15 \mathrm{GeV}$. One of the interesting physics motivation at ELPH is to study a penta-quark candidate, $N^{*}(1670)$ resonance, which couples to $n \eta$ channel and not to $p \eta$. The details of the study on $N^{*}(1670)$ is reported by T. Ishikawa in this issue.

SPring-8/LEPS provides a highly polarized photon beam of $\sim 2$ Mcps which is produced by the backward-Compton scattering (BCS) process. A photon beam with maximum energy up to $2.4 \mathrm{GeV}$ is available using 355-nm laser. A higher energy photon beam up to $2.9 \mathrm{GeV}$ can be produced using $266 \mathrm{~nm}$ laser with $\sim 0.5 \mathrm{Mcps}$ [四]. At the LEPS energy, a $s \bar{s}$ quark pair can be produced from a photon, and thus photoproduction of $\phi$-meson and hyperons has been studied intensively. A new GeV photon beamline (LEPS2) has been constructed at SPring-8 in 2013. We have successfully observed the first beam, and the commissioning experiment has started. In this article, recent results from LEPS and status of LEPS2 are described.

\section{Kaonic nuclei search in the $\gamma d \rightarrow K^{+} \pi^{-} X$ reaction}

The exotic nuclei which contain particles other than nucleons are fascinating objects in the nuclear and hadron physics. The exotic particle changes the structure of the nucleus and the property of that particle may change in the nuclear medium. Hyper nuclei which contain hyperons as components have been investigated intensively. The hyperon in the nucleus plays interesting roles such as glue-like role of $\Lambda$ hyperon in ${ }_{\Lambda}^{9} \mathrm{Be}$. The exotic nuclei which contain other than hyperons, $\omega, \eta$ or kaon, have been searched for but not been established so far. Recently, nuclei which contain an anti-kaon, kaonic nuclei, have been studied with great interest since the prediction by Y. Akaishi and T. Yamazaki [2]. The experimental search for kaonic nuclei is in the controversial situation. FINUDA experiment at DAФNE [B] and DISTO experiment at SATURNE [4] and OBELIX experiment at LEAR-CERN [5] reported positive results but their measurements of the binding energy or width are not consistent. Another search experiment at HADES reported negative results [ 6 ]. Thus, the existence of the kaonic nucleus is not established, and further experimental searches are awaited. Here, we report the first result of search for a kaonic nucleus, $K^{-} p p$, using a photon beam [四].

The experiment has been carried out at SPring-8/LEPS using a photon beam of $E_{\gamma}=1.5-$ $2.4 \mathrm{GeV}$. We employed a liquid deuteron target, and a peak structure was searched for in the missing mass of the $\gamma d \rightarrow K^{+} \pi^{-} X$ reaction. The LEPS magnetic spectrometer detected a $K^{+} \pi^{-}$pair which 
was produced at forward angles, where the $t$-channel reaction is dominant. In the photon induced reaction, a $K^{-}$can be exchanged in the $t$-channel, while it is forbidden in the meson induced reaction due to the parity conservation. Thanks to the high intensity photon beam at LEPS, we

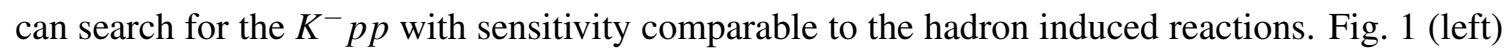
shows the missing mass spectrum of the $\gamma d \rightarrow K^{+} \pi^{-}$reaction. Closed circles show the data and the inset shows the spectrum in the region where the $K^{-} p p$ signal is expected. The dashed line is an eye guide. We can see peaks around $1.9 \mathrm{GeV} / \mathrm{c}^{2}, 2.1 \mathrm{GeV} / \mathrm{c}^{2}, 2.2 \mathrm{GeV} / \mathrm{c}^{2}$ and $2.5 \mathrm{GeV} / \mathrm{c}^{2}$, which correspond to neutron, $\Lambda, \Sigma$ and hyperon resonances, respectively. No peak structure was observed in the $K^{-} p p$ signal region. Fig. $\mathbb{W}$ (right) shows the upper limit of $K^{-} p p$ production as a function of the mass of $K^{-} p p$ for several assumptions of width. The details of the analysis are reported by A.O. Tokiyasu in this issue.
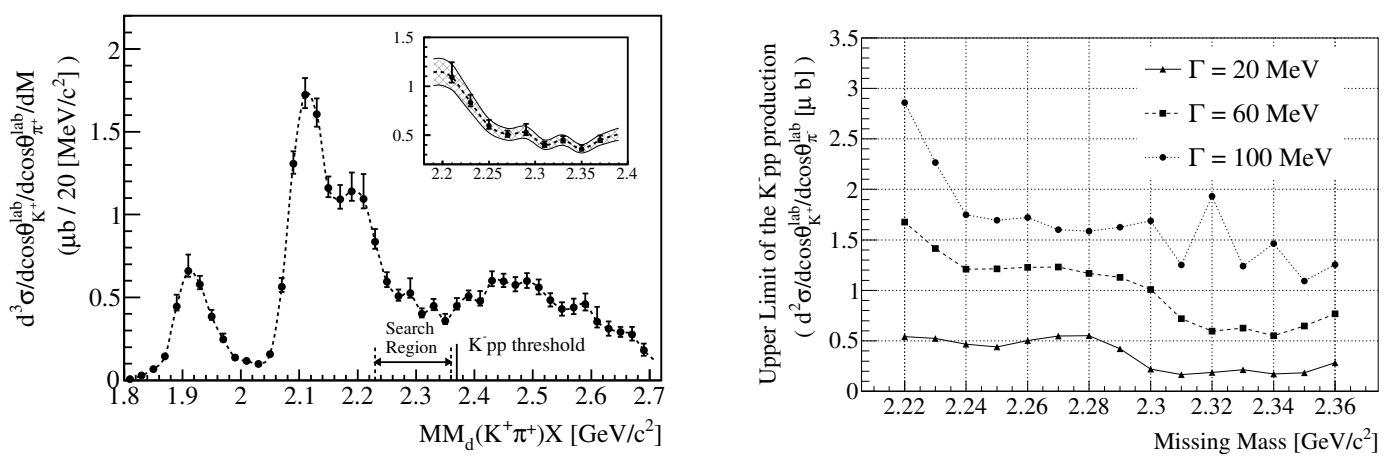

Figure 1: Left: missing mass spectrum of $\gamma d \rightarrow K^{+} \pi^{-}$reaction. Right: upper limit of $K^{-} p p$ production as a function of the mass of $K^{-} p p$ for several assumptions of width.

\section{3. $\Theta^{+}$search in the $\gamma d \rightarrow K^{+} K^{-} p n$ reaction}

Evidence of a penta-quark state $\Theta^{+}$which consists of $u u d d \bar{s}$ quarks has been reported by the LEPS collaboration in 2003 [ [8]], but its existence is still controversial. The DIANA collaboration observed a peak structure in the $K^{+}$Xe reaction [Q, 四, 四] while the Belle collaboration did not find any peak using the events where $K^{+}$'s hit the detector [U2]. The CLAS collaboration also searched for $\Theta^{+}$in $\gamma d \rightarrow K^{+} K^{-} p n$ reaction by measuring $K^{+} K^{-}$and proton in the final state, however no peak structure was observed [[13]]. The LEPS collaboration has obtained an additional positive result with a $\sim 5 \sigma$ significance from data collected in 2002-2003 [U4]]. Here, the $\gamma d \rightarrow$ $K^{+} K^{-} p n$ reaction has been analyzed by identifying a quasi-free nucleon reaction inclusively from a missing mass $M M_{N}\left(\gamma, K^{+} K^{-}\right)$and by assuming a spectator nucleon has the minimum momentum. This assumption is good approximation to minimize a Fermi motion effect. An photon-energy dependent $\phi$ meson-exclusion cut improves signal-to-noise (S/N) ratios of the $\Lambda(1520)$ and the $\Theta^{+}$ in invariant mass distributions $M\left(K^{-} p\right)$ and $M\left(K^{+} n\right)$, respectively. Since the second publication on the $\Theta^{+}$search, we took 2.6 times larger data in 2006-2007 using the almost same experimental setup. The calibration methods and analysis procedure were same as the analysis of 2002-2003 data. The quality of the new data was confirmed under the blind analysis condition in which the $\Theta^{+}$signal region was masked. Recently, we unmasked the $M\left(K^{+} n\right)$ spectrum including the 
signal region. A significance in the new data becomes lower, resulting in inconsistency with the previous analysis. In order to investigate the reason of the inconsistency, we improved our analysis to separate quasi-free neutron and proton contributions exclusively.

We employed two alternative analyses methods to separate the events in which an incident photon hits a proton or a neutron in a deuteron. Firstly, a trigger plastic scintillator which is located just downstream of the target is utilized to measure the energy deposit of $K^{+} K^{-}$only or $K^{+} K^{-} p$ (dE/dx-based analysis). The energy deposit of each particle is corrected depending on its momentum and angle, and the events in which only $\mathrm{K}^{+} K^{-}$hit the trigger scintillator are collected by selecting the events with small energy deposits (proton rejected events). By selecting proton rejected events, we can enhance the quasi-free $\gamma n \rightarrow K^{+} K^{-} n$ reaction where we expect $\Theta^{+} K^{-}$ production. On the other hand, it is forbidden in the $\gamma p \rightarrow K^{+} K^{-} p$ reaction due to the charge conservation. Red points in Fig. $\square$ show a $M\left(K^{+} n\right)$ spectrum for the proton rejected events, where a combined sample of the 2002-2003 and 2006-2007 data was used. A clear signal peak can be seen around $1.53 \mathrm{GeV} / \mathrm{c}^{2}$. Here the z-position of a reaction vertex is required to be close to the trigger scintillator, so that a proton tagging efficiency is raised up to $\sim 90 \%$. Another exclusive analysis is carried out by estimating the quasi-free proton contribution based on MC simulations (MC-based analysis). Photoproduction of $\phi p$, non-resonant $K^{+} K^{-} p$ and $K^{+} \Lambda(1520)$ are simulated after adjusting photon-energy and angular dependence of the yield using the $K^{+} K^{-} p$ events. A histogram in Fig. $\square$ shows a $M\left(K^{+} n\right)$ spectrum after subtracting the quasi-free proton contribution, estimated by the MC simulations. The two spectra made by independent exclusive analysis agree with each other, suggesting a signal peak structure. The study on the background shape and statistical significance is underway. In addition, in order to enhance the proton rejection efficiency, we started further data taking using a larger trigger counter.

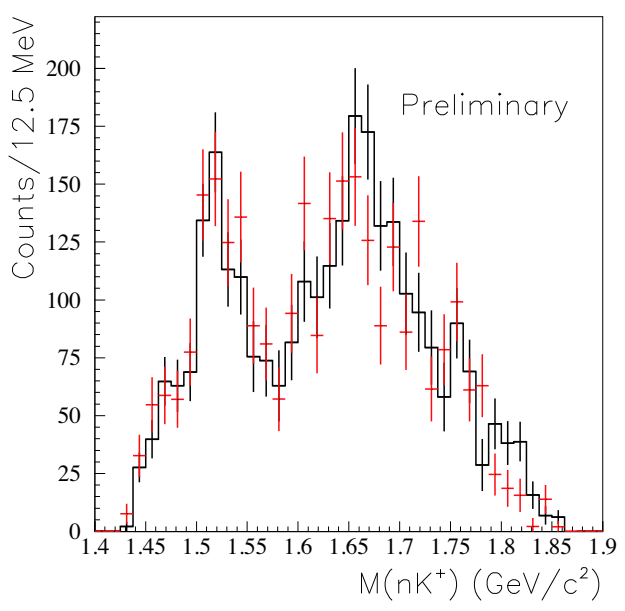

Figure 2: Invariant mass spectra obtained by exclusive analysis using $\mathrm{dE} / \mathrm{dx}$-based analysis (red points) and MC-based analysis (black histogram). 


\section{LEPS2 experiment}

A new GeV photon beamline has been constructed at SPring-8 (LEPS2) utilizing a long straight section of the electron storage ring, which has an excellent beam emittance. Thanks to the quality of the electron beam, the angular divergence of BCS photon beam is small enough to be guided to $150 \mathrm{~m}$ downstream and to be incident on the target. A new experimental hall has been constructed out of the SPring-8 storage ring building, where large solid angle detectors can be installed. The intensity of the photon beam is also upgraded to be 10 times higher than that of LEPS by injecting four lasers simultaneously and by focusing the laser to match to the shape of the $8-\mathrm{GeV}$ electron beam. We have successfully observed the first beam in January 2013. Fig. [3] (a) shows energy spectra of the photon beam, which were measured using an electromagnetic calorimeter. The black and blue histograms show spectra with and without the laser injection. A clear Compton spectrum (black histogram) which shows the Compton edge at $2.4 \mathrm{GeV}$ can be seen with the laser injection, while a low intensity Bremsstrahlung spectrum (blue histogram) is visible without the laser, which is produced by the interaction of the electron beam with the gas in the storage ring. The spatial distribution of the BCS photon beam in the experimental hall is shown in Fig. (B] (b). The beam size was confirmed to be quite small at the target which is located $\sim 150$ m downstream from the BCS interaction point.
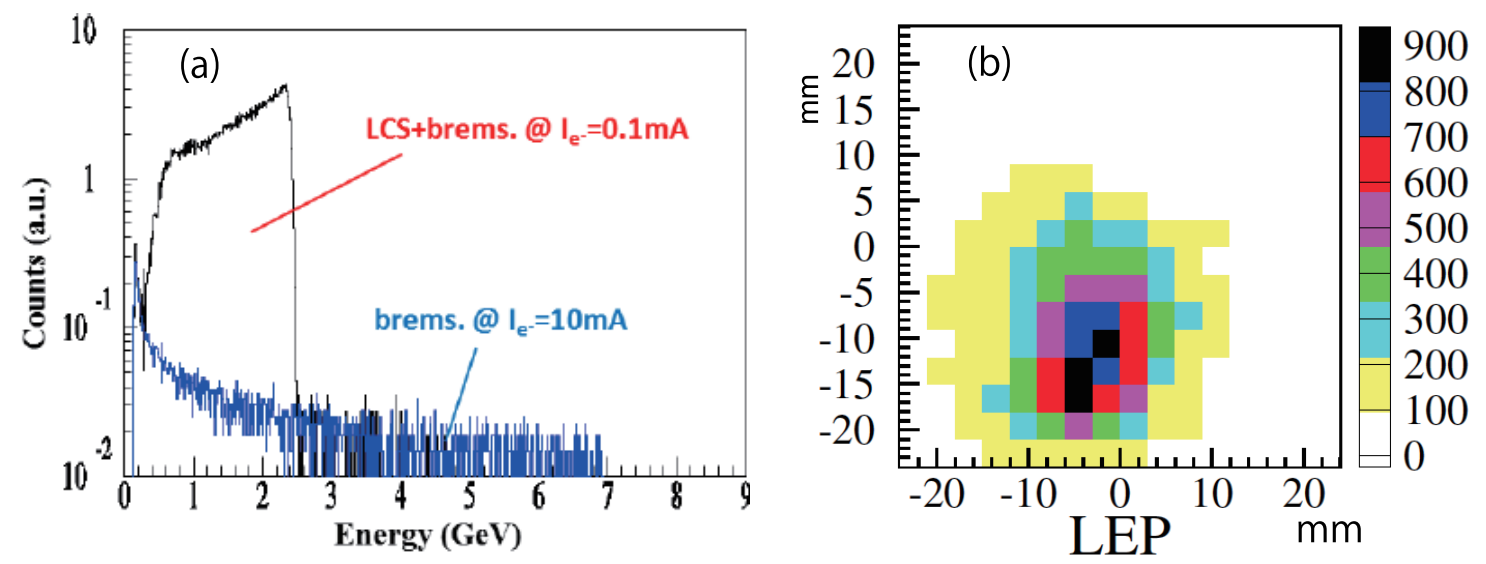

Figure 3: (a) Energy spectra of the photon beam with and without the laser injection. (b) Spatial distribution of the BCS photon beam in the experimental hall.

Two different experiments have been proposed at LEPS2, one is the experiment using a photon detector, BGO-EGG, and the other is the large solenoid magnetic spectrometer. The BGO-EGG has been developed by the ELPH group who has many experiences on electromagnetic calorimeters. The BGO-EGG consists of $1320 \mathrm{Bi}_{4} \mathrm{Ge}_{3} \mathrm{O}_{1} 2$ (BGO) crystals which are arranged as 'EGG'-shape. The polar-angle coverage is $24^{\circ}<\theta<144^{\circ}$ and thickness of each crystal is $\sim 20$ radiation lengths as shown in the left panel of Fig. 目. The energy resolution was evaluated to be $1.3 \%$ at $1 \mathrm{GeV}$ using a positron beam at ELPH. A cylindrical drift chamber and a planar drift chamber are installed inside and downstream of the BGO-EGG, respectively, to measure directions of charged particles. In addition, a high resolution time-of-flight counter which consists of resistive plate chambers is located $\sim 12 \mathrm{~m}$ downstream from the target, which can measure the energy of a forward-going 
proton with $\sim 20 \mathrm{MeV}$ resolution (r.m.s.) at the $2 \mathrm{GeV}$ energy. The commissioning run using a part of the detector system has started in late 2013, and the physics run will start from spring in 2014. Various meson photoproduction from a liquid hydrogen or nuclear targets will be measured using the linearly polarized beam.

The right panel of Fig. 1 shows the LEPS2 solenoid magnetic spectrometer. The solenoid magnet which was used at the kaon rare-decay experiment at Brookhaven National Laboratory (BNL-AGS, E787/E949). The inner diameter and length are $2.96 \mathrm{~m}$ and $2.22 \mathrm{~m}$, respectively, and the magnetic field of $1 \mathrm{~T}$ is applied. The barrel electromagnetic calorimeter has also been shipped from BNL to be used together with the solenoid magnet at LEPS2. The detectors for charged particle tracking and particle identification (PID) are newly constructed. A time projection chamber and four drift chambers are employed for the tracking. In order to identify low momentum kaons, a high resolution time-of-flight counter consists of resistive plate chambers are used. For the PID of high momentum particles, aerogel Cherenkov counters and time-of-propagation counters [[5]] will be developed. The one of the main physics purpose is to study the $K^{-} \Theta^{+}$production from deuteron. The LEPS experiment measured $\Theta^{+}$signal by detecting a $K^{-}$scattered at a forward angle, on the other hand, the CLAS experiment covers the large polar angle region and did not observe the signal. The LEPS2 spectrometer can detect $K^{-}$'s from $7^{\circ}$ to $\sim 110^{\circ}$ and investigate the $\Theta^{+}$in the invariant mass of $K_{S}^{0}$ proton. If strong angular dependence of $K^{-} \Theta^{+}$production cross section is observed, it will solve the controversial situation between the LEPS and CLAS results. Detector development is underway, and physics run will start in near future.
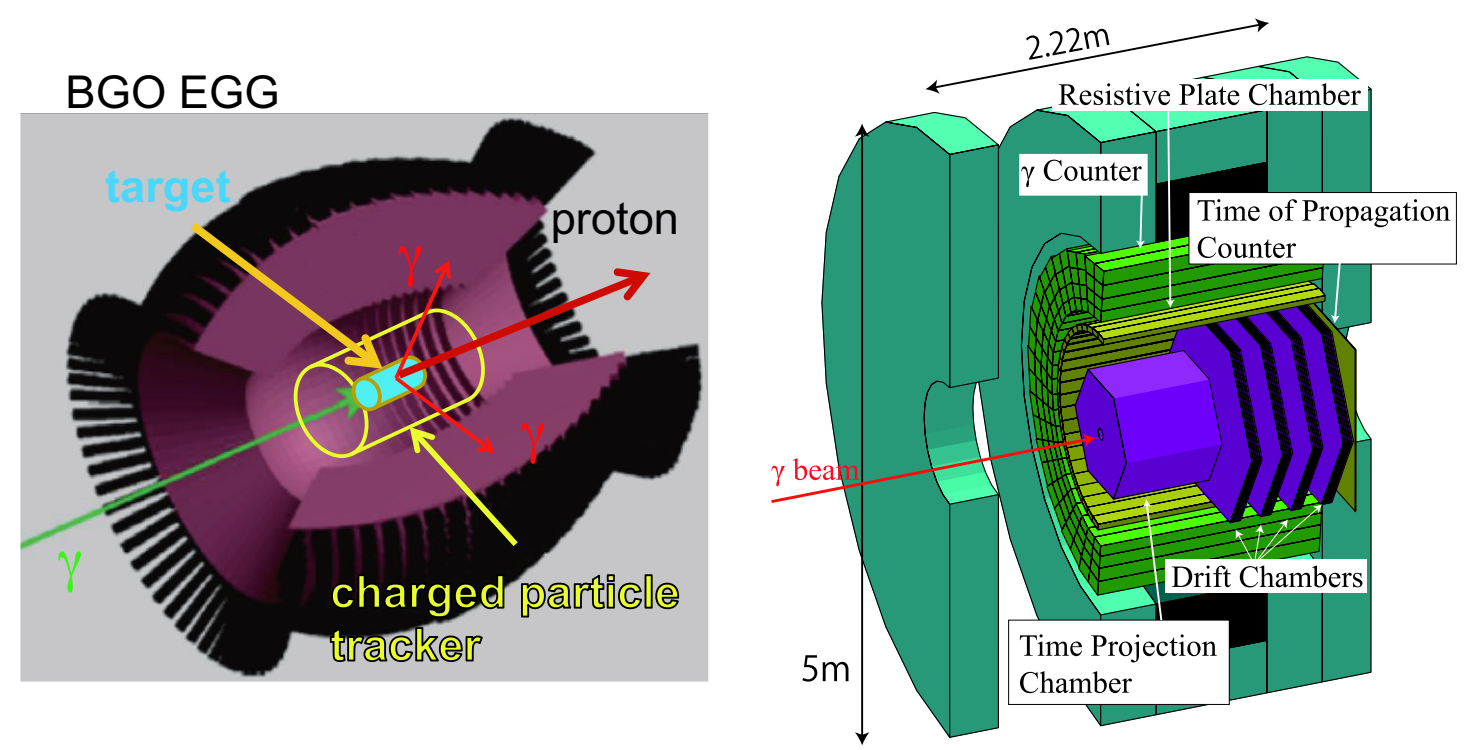

Figure 4: Schematic drawings of the BGO-EGG detector (left) and the LEPS2 solenoid magnetic spectrometer (right).

\section{References}

[1] N. Muramatsu et al. Nucl. Inst. Methods. A 737184 (2014).

[2] Y. Akaishi and T. Yamazaki, Phys. Rev. C 65, 044005 (2002). 
[3] M. Agnello et al. Phys. Rev. Lett. 94212303 (2005).

[4] T. Yamazaki et al. Phys. Rev. Lett. 104132502 (2010).

[5] G. Bendiscioli et al. Eur. Phys. J., A40 11 (2009).

[6] L. Fabbietti et al. Nucl.Phys., A914 60 (2013).

[7] A.O. Tokiyasu et al. Phys. Lett. B728 616 (2014).

[8] T. Nakano et al. Phys. Rev. Lett. 91, 012002 (2003).

[9] V.V. Barmin et al. Phys. Atom. Nucl. 661715 (2003).

[10] V.V. Barmin et al. Phys. Atom. Nucl. 7035 (2007).

[11] V.V. Barmin et al. arXiv:1307.1653 [nucl-ex] .

[12] R. Mizuk et al. Phys. Lett. B632 173 (2006).

[13] B. McKinnon et al. Phys. Rev. Lett. 96, 212001, (2006).

[14] T. Nakano et al. Phys. Rev. C. 79, 025210 (2009).

[15] M. Akatsu et al. Nucl. Inst. Methods. A 440, 124 (2000). 